\title{
Implication of COVID-19 on Post-Secondary Students' Mental Health: A Review
}

\author{
Carly Sillcox ${ }^{1}$
}

${ }^{1}$ Faculty of Science, McGill University

\section{Correspondence}

Carly Sillcox

Email: carlysillcox@gmail.com

\section{Publication Date}

March 4, 2022

MJM 2022 (20) 25

https://doi.org/10.26443/mjm.v20i1.922

\section{ণ் McGill \\ Journal of Medicine}

\author{
www.mjmmed.com
}

\section{c) (i) (2) (2)}

This work is licensed under a Creative Commons BY-NC-SA 4.0 International License.

\section{A BSTRACT}

Introduction: Nearing two nears into the current pandemic, COVID-19 is recognized worldwide for its devastating physical effects, with mandatory restrictions implemented to prevent the transmission of SARS-CoV-2. However, the world is only beginning to understand the pandemic's mental and social side effects. As such, current research on consequential mental health from COVID19 is still novel, and there is much more to be learned concerning the long-term psychological effects and damage from the pandemic.

Discussion: The combination of online learning and social isolation due to COVID-19 has affected post-secondary students across North America as it relates to their overall well-being and mental health. Researchers have aimed to examine the psychological impact on students' mental health, primarily through cross-sectional studies and self-reported surveys.

Conclusion: Studies have determined that COVID-19 has increased mental health symptoms such as depression, anxiety, PTSD, as well as increased feelings of isolation, loneliness, and fatigue. Furthermore, drinking and substance use, poor sleeping patterns, and screen time have risen as a result of the ongoing pandemic.

Relevance: These findings call for post-secondary institutions, health care providers, and governments to prioritize the mental health of future generations while providing support and intervention programs. Future research should focus on further investigating COVID-19's long-term effects on the mental health of post-secondary students and exploring prevention methods.

\section{KEYWORDS}

COVID-19, Mental health, Quarantine, Universities, Students

\section{1 | INTRODUCTION}

The ongoing COVID-19 global pandemic has given rise to a new norm of societal living, consisting of a lifestyle predominately based on working, studying, and living from home. Governmental restrictions such as quaran- tine, lockdown, and curfew have forced individuals to stay home in an effort to prevent the spread of SARSCoV-2. Globally, the public has adapted to the physical effects of the virus by wearing masks, practicing social distancing, and performing handwashing/sanitization. However, society has greatly overlooked the mental 
and social effects of COVID-19. To date, researchers have conducted minimal in-depth studies on individuals' mental health as a side effect of the COVID-19 pandemic. In particular, the young adult population has been obligated to transform their learning and living methods over the past year. As a result, the well-being and mental health of higher education students have been severely compromised. In recent months, numerous studies of the pandemic's psychological impact on university and college students have been undertaken. Thus far, the research reveals that the COVID-19 public health crisis has greatly heightened mental health risk factors, including social isolation, unemployment, insecurity, and instability. (1) Also, feelings such as loss of freedom, separation from loved ones, uncertainty, and boredom have significantly contributed to the decline in youth mental health. (2) Furthermore, some studies show increased stress, anxiety, and depression symptoms in post-secondary participants. (1) Despite the newly found research regarding the declining mental health of higher education students due to COVID19 , much work remains to be done. Particularly, preventing stigmatization, raising awareness, and determining the pandemic's long-term effects on students are of utmost importance. In this review, topics including but not limited to motivation loss, isolation, depression, anxiety, drinking, substance use, screen time, sleep patterns, self-harm, and suicide will be addressed in the context of post-secondary students' mental health during COVID-19. The findings from this review will help to motivate future research on COVID-19's long-term mental and social impacts and encourage institutional action by higher education leaders to preserve the wellbeing of students.

\section{2 | METHODS}

This review conducted database searches on youth mental health during COVID-19, through Google Scholar, PubMed, QJM, and APA Psyclnfo. Inclusion criteria included North American studies conducted between January 2020 - July 2021 that were published in En- glish. Additionally, equal emphasis was placed on peer-reviewed articles, psychiatry research, and credible news articles that were relevant to student mental health. Exclusion criteria included studies conducted prior to COVID-19, with an exception being studies comparing the state of mental health before and during the pandemic. The databases were searched using variations of the following terms (and their synonyms): "COVID-19" OR "coronavirus" OR "pandemic" / "young adult" OR "adolescent" OR "youth" OR "teenager" / "student" OR "post-secondary" OR "university" OR "college" / "lockdown" OR "quarantine" OR "curfew" / "mental health" OR "psychological impact" / "social isolation" OR "loneliness" / "depression" OR "anxiety" OR "PTSD" OR "stress" / "North America" OR "Canada" OR "U.S." Results included a combination of peer-reviewed journals and abstracts. More than 100 studies were surveyed, with the final review limited to 12 studies highlighting the in-depth findings of student mental health during the pandemic. The final review included 31 publications, of which 12 were peer-reviewed journals, 5 were psychiatry research pieces, 5 were university published articles, 5 were relevant statistical sources, and 4 were important opinion news articles.

\section{3 | DISCUSSION}

\section{1 | A World of Change}

The start of the pandemic uprooted post-secondary students across North America from their socially fulfilling school environments, and the vast majority were sent home to finish their semester using an entirely new platform: Zoom. (3) On top of the uncertainty surrounding the rising spread of COVID-19, students were expected to make a major transition in terms of how they learned and managed routine daily tasks. Carlos Fuentes, a journalist for The Beacon Newspaper at the University of Portland, writes, "Zoom class backgrounds have become a collage of different houses, varying time zones and a range of outside stressors that go unseen on camera." (3) The years 2020-2021 will be remembered in history not only for a global pandemic, but also for other over- 
whelming societal and environmental issues that were brought to the forefront, including climate disasters, systemic racism, and a tumultuous state of political affairs in the U.S. During an extended period of isolation, in addition to profound social, political, and economic unrest, recent studies have recognized young adults as the most vulnerable group for depression and anxiety during the pandemic. (4) Moreover, mental health professionals are becoming "increasingly alarmed about the deteriorating mental state of young people, who they say have been among the most badly affected by a world with a foreshortened sense of the future" due to the COVID-19 pandemic. (5)

\subsection{Fatigue and Loss of Motivation}

Online learning and its unforeseen consequences were not an easy adjustment for most higher-level education students, in turn producing a loss of motivation and inspiration while increasing overall fatigue. (3) Studies show that in-person school environments are crucial for promoting academic motivation and social development. (6) As a Huffington Post article from February 2021 details, "From a spotty Wi-Fi connection, to broken links, a distracting work environment and poor communication from professors - virtual education comes with a major learning curve." (7) Audio delays and low-resolution calls require extended attention. (8,9) Without the support and engagement of in-person learning, students struggle to make it through their online classes and become susceptible to 'Zoom Fatigue.' (8,9) For most students, video calls expend more energy and require more focus than face-to-face interactions as non-verbal cues, tone/pitch of voice, and body language are more challenging to process online. $(9,10)$ "Our minds are together when our bodies feel we're not. That dissonance, which causes people to have conflicting feelings, is exhausting. You cannot relax into the conversation naturally," says Gianpiero Petriglieri, an associate professor at Insead University. (10)

\subsection{Isolation and Loneliness}

Moreover, the sudden transition to online learning triggered a strong sense of disconnect for students across North America. The support systems of university and college students largely vanished beginning in the spring of 2020 on account of COVID-19's isolation requirements. (4) A question posed by many schools and universities was how to "nurture social connection in the virtual, inherently disconnected environment of the pandemic?" (4) Students often experience feelings of loneliness and isolation without their friends for extended periods; therefore, friends serve as mental health protective factors. (3) The University of Oxford conducted a virtual longitudinal study, titled Achieving Resilience During COVID-19 (ARC), with findings highlighting that young people are lonelier in lockdown than their parents. (11) Likewise, in a self-response survey of 2,500 U.S. college students, $80 \%$ of participants said they would be more educationally successful if living with friends rather than at home. (3) As a Tulane University paper on loneliness and isolation in COVID-19 states, "Social support plays a key role in well-being, yet one of the major preventative efforts for reducing the spread of COVID-19 involves social distancing." (12) Even though social support is a known coping mechanism in times of crisis, its benefits are limited during the pandemic due to social distancing and other governmental restrictions implemented. (12) Additionally, "isolation has also disrupted the usual teenage transition, when young people move from belonging to their family to belonging to their peers," Dr. Vermeiren, a psychiatrist at Leiden University Medical Center, explains. (5) This developmental concern causes feelings of emptiness and loneliness, consequently leading students into a state of despair. (5)

\section{4 | Depression, Anxiety, and PTSD}

Faced with numerous restrictions, uncertainty, and a roller coaster of emotions, university and college students' mental health has declined, as evidenced by increased rates of depression, anxiety, and PTSD symptoms. $(13,14)$ Researchers from McGill University and 
the University of Toronto proclaim that "the psychological impacts will be as significant as the physical health impacts from COVID-19, particularly for vulnerable populations." (13) Throughout several self-reported cross-sectional mental health studies, the prevailing consensus is that post-secondary participants' levels of worry, grief, depression, anxiety, and PTSD have increased during COVID-19. (13,14) According to Statistics Canada, younger Canadian participants exhibited more pronounced moderate to severe symptoms of anxiety than older participants throughout the pandemic. (15) Moreover, February 2021 research from Toronto's SickKids Hospital reveals that "greater stress from social isolation, including both the cancellation of important events and the loss of in-person social interactions, was strongly associated with mental health deterioration." (16)

\subsection{Mental Health Prior to Pandemic}

Among several studies detailing increased mental health and psychological concerns throughout the pandemic, $(17,18)$ an exception is a North American longitudinal study with a sample size of 773 post-secondary students - with and without pre-existing mental conditions - examining the differences in mental health responses from May 2019 (pre-pandemic) and May 2020 (pandemic). (13) Although researchers expected participants with pre-existing mental health symptoms to have heightened psychological distress during the pandemic, this hypothesis was disproved. (13) Instead, this group of participants had similar or better mental health during the pandemic. (13) Contrarily, the student participants with no mental health concerns pre-COVID19 developed significant psychological distress during the pandemic. (13) These results shed light on social isolation's impact on individuals previously presenting with no mental health concerns. However, at this time there is no affirmative explanation for the unexpected improved mental health of pre-pandemic mental health diagnosed participants. (13)

\subsection{Drinking and Substance Use}

A cross-sectional anonymous online survey of 1000 youth participants, aged 18-35, studied the correlation of loneliness during COVID-19 with depression/anxiety and alcohol/drug use. (19) Researchers found substantial increases in mental health concerns and substance use due to the pandemic. (19) On top of rising stress and anxiety levels, young adults tend to follow the example set by adults, which is another contributing factor of substance use during COVID-19. (20) As mentioned in a Drug Free Kids Canada article, "It is important for parents to be aware that they may have increased their own substance use to deal with their stress, and as a consequence, their kids may consider that using substances is an appropriate way to cope with their uncomfortable feelings." (20) As reported by a Nanos Research poll summary, a quarter of Canadian participants aged 3554 increased their drinking patterns during COVID-19 due to stress, boredom, and lack of a regular schedule. (21) As this participant age group includes many postsecondary students' role models, this statistic highlights the mirror effect of substance use as a coping mechanism on youth during troubling times.

\section{7 $\quad$ Screen Time}

Excessive screen time is another negative aspect of the pandemic on higher education students' mental health. From keeping up with and communicating through online learning, social media, news/entertainment, and loved ones, young adults have spent much longer staring at devices and sitting in front of screens throughout the lockdown than ever before. (22) According to a current review of screen time and COVID-19, a survey recorded that the pandemic caused a $50-70 \%$ increase in internet use, with $50 \%$ of that time spent engaging on social media. (23) Research from an online crosssectional study of 932 students in the United Kingdom shows a correlation between daily screen time and mental health concerns, as measured using the Beck Anxiety Inventory (BAI) and the Beck Depression Inventory (BDI) tools, reflecting higher cases of anxiety and depression, 
respectively. (22) This study accounted for potential confounding factors, and its results are translatable to North American students. (22) Experts suggest 'digital detoxes' and unplugging for hours or days, which typically result in increases in productivity, creativity, connections with loved ones, quality sleep, and decreases in aches/pains. (25) Harvard Pilgrim Health Care claims that "you can feel the physiological effects almost immediately when initiating a digital detox." (25)

\section{8 | Sleep Patterns}

Given that as screen use, particularly before falling asleep, negatively affects sleep quality, Harvard Health shows that there is also a strong correlation between sleep and mental health concerns. (26) Sleep deprivation is known to impact one's mental health and psychological state. (26) Likewise, individuals with mental health concerns are more at risk for sleep disorders such as insomnia. (26) For instance, studies often compare depression, anxiety, and PTSD with sleep patterns and sleep disorders. (26). Multiple studies conclude that most patients who categorized with one or more mental health concerns also experience sleeping problems or sleeping disorders. $(26,27)$ Generally speaking, sleep problems increase the risk of developing mental health symptoms such as depression. (27) As a McGill University sociology student, Erika MacKenzie, explains in her opinion article speaking on behalf of the student population nationwide, "In conjunction with being drained, the lack of sleep made me more anxious and depressed, further inhibiting my academic performance." (7) Overall, "exposure to stimulating content, mobile phone overuse and phone addiction contribute to hyper arousal in prebedtime period and poor sleep quality," which cause excessive daytime sleepiness and contribute to mental health concerns. (27)

\section{9 | Self-Harm and Suicide}

With severe isolation, loneliness, stress, and negative emotions due to COVID-19, some post-secondary students face alarming consequences, including self-harm and suicide. (4) Throughout the pandemic, youth suicide has become the second leading cause of death in individuals aged 15-24. (4) Research from Providence St. Joseph Health, a non-profit healthcare system in various U.S. states, expresses that citizens are greatly at risk of deaths of despair: deaths from drugs, alcohol, and suicide. (28) As quoted in their 'Projected Deaths of Despair from COVID-19' abstract, "Deaths of despair have been on the rise for the last decade, and in the context of COVID-19, deaths of despair should be seen as the epidemic within the pandemic." (28) Researchers warn of "a grim picture of the struggle with lockdown isolation - a 'mental health pandemic' that should be treated as seriously as containing the coronavirus." (5) Throughout the U.S., schools are announcing severe rises in self-harm and suicides. (4) Notably, "it is worth remembering that more young people will die from suicide and road traffic accidents than COVID-19 this year." (11) In essence, students feel that they have sacrificed more than a year of their youth to protect their elders, and in the process, they have experienced devastating side effects. (11)

\subsection{0 | Available Resources}

With the accumulation of complex challenges faced by higher education students, school counsellors should not be the only resource available; instead, postsecondary institutions have an ongoing obligation to monitor their students' mental health, both before and after returning to campus. As the return to on-campus learning becomes a reality for upcoming semesters, post-secondary institutions must take responsibility for their student body and prioritize the mental health of their students. The Mental Health Commission of Canada reports the following:

Three out of every four mental health problems have been first diagnosed between the ages of 16 and 24, when many are in or just out of post-secondary education. In 2020, the COVID-19 pandemic heightened many of the issues students were already facing, making it even more challenging for institutions to support them. (29)

It is imperative for higher education leaders to ac- 
knowledge such obligations and to initiate changes on campus, both remotely and in person. Post-secondary students should have multiple methods and resources at their disposal to discuss their well-being, mental health, and other concerns. School counsellors are indispensable; however, "they can't be the only office on campus responsible for students' mental health. Administrators and faculty and staff members all have a role to play in ensuring that students are not only surviving but also thriving." (30) Educational institutions have a duty to perform as it cannot be expected that such a fragile age group can simply return to campus as if COVID19 never happened. Thus, as the real impact of COVID19 makes itself apparent, there is an institutional obligation to monitor and provide ongoing outreach and counselling services for as long as the demand warrants.

\section{4 | CONCLUSION}

\section{1 | Future Research}

The COVID-19 pandemic has touched hundreds of millions of people physically and taken the lives of over 5 million individuals, (31) yet the resultant mental and social aspects cannot be ignored. Post-secondary students across North America have experienced incredibly damaging effects to their mental health triggered by social isolation and online learning. This crisis should not be taken lightly as it becomes a growing concern the longer the pandemic continues, and the longer students are kept away from their normal social lives and in-person education. Consequently, long-term mental health concerns arise, including depression, anxiety, PTSD, substance abuse, screen-overuse, and sleep issues. "Education changes the way we perceive the world and behave in relation to others, and this affects our brain directly. The consequences for youth development in the years to come could be vast, with impacts likely on self-control, social competence and logical deduction amongst other cognitive abilities." (11) Higher education institutions are well aware of the mental health damage over the past year. This early research is fuelling their efforts to get students back to in-person learning as quickly as possible without circumventing safety. Nonetheless, there is an urgent need for further research on COVID-19's long-term impact on the mental health of post-secondary students. Then, armed with facts, prevention and intervention by schools, communities, and government must be prioritized to preserve the well-being of our youth.

\section{2 | Acknowledgements}

The author would like to thank professor Dr. Noreen Bider from McGill University for her continual support and encouragement during the writing process. In addition, the author would like to thank the McGill Journal of Medicine and its editorial team for offering an opportunity for students to experience a peer-review process and publish something meaningful to them and their community.

\section{REFERENCES}

1. Johnson HR. Position Paper: The Impact of COVID19 on Mental Health [Internet]. Psychiatry Advisor. 2021 [cited 2021 March 12]. Available from: https://www.psychiatryadvisor.com/home/topics/generalpsychiatry/position-paper-the-impact-of-covid-19-on-mentalhealth/

2. Javed B, Sarwer A, Soto E, Mashwani Z. The coronavirus (COVID19) pandemic's impact on mental health. The International Journal of Health Planning and Management. 2020;35(5):993-996. [cited 2021 Feb 22]. Available from: https://doi.org/10.1002/hpm.3008

3. Fuentes $\mathrm{C}$. Zoom fatigue and all-nighters: Online learning takes a toll on students' mental health [Internet]. The Beacon. 2021 [cited 2021 March 18]. Available from: https://www.upbeacon.com/article/2020/10/zoom-fatigue-andall-nighters-online-learning-takes-a-toll-on-students-mentalhealth

4. Becker M. Educators are key in protecting student mental health during the COVID-19 pandemic [Internet]. Brookings Institution. 2021 [cited 2021 March 17]. Available from: https://www.brookings.edu/blog/brown-centerchalkboard/2021/02/24/educators-are-key-in-protectingstudent-mental-health-during-the-covid-19-pandemic/

5. Kwai I, Peltier E. 'What's the Point?' Young People's Despair Deepens as Covid-19 Crisis Drags On [Internet]. The New York Times. 2021 [cited 2021 March 18]. Available from: https://www.nytimes.com/2021/02/14/world/europe/youth- 
mental-health-covid.html

6. Stringer H. Zoom school's mental health toll on kids [Internet]. American Psychological Association. 2021 [cited 2021 March 16]. Available from: http://www.apa.org/news/apa/2020/10/onlinelearning-mental-health

7. MacKenzie E. Don't Tell Me The Isolation Of Online Learning Is Worth A Full University Tuition [Internet]. HuffPost Canada. 2021 [cited 2021 March 17]. Available from: https://www.huffingtonpost.ca/entry/online-learning-universitytuition_ca_602591f1c5b680717ee67792

8. Schroeder R. Zoom Fatigue: What We Have Learned [Internet]. Inside Higher Ed. 2021 [cited 2021 March 20]. Available from: https://www.insidehighered.com/digital-learning/blogs/onlinetrending-now/zoom-fatigue-what-we-have-learned

9. Williams N. Working through COVID-19: 'Zoom' gloom and 'Zoom' fatigue. Occupational Medicine. 2021;71(3):164-164. [cited 2021 Aug 31]. Available from: https://doi.org//10.1093/occmed/kqab041

10. Jiang $M$. The reason Zoom calls drain your energy [Internet]. BBC News. 2021 [cited 2021 March 20]. Available from: https://www.bbc.com/worklife/article/20200421-whyzoom-video-chats-are-so-exhausting

11. Townsend E. Debate: The impact of school closures and lockdown on mental health in young people. Child and Adolescent Mental Health. 2020;25(4):265-266. [cited 2021 March 17]. Available from: https://doi.org/10.1111/camh.12428

12. Saltzman L, Hansel T, Bordnick P. Loneliness, isolation, and social support factors in post-COVID-19 mental health. Psychological Trauma: Theory, Research, Practice, and Policy. 2020;12(S1):S55-S57. [cited 2021 Feb 22]. Available from: https://doi.org/10.1037/tra0000703

13. Hamza C, Ewing L, Heath N, Goldstein A. When social isolation is nothing new: A longitudinal study on psychological distress during COVID-19 among university students with and without preexisting mental health concerns. Canadian Psychology. 2021;62(1):20-30. [cited 2021 Feb 22]. Available from: https://doi.org/10.1037/cap0000255

14. Conrad R, Hahm H, Koire A, Pinder-Amaker S, Liu C. College student mental health risks during the COVID-19 pandemic: Implications of campus relocation. Journal of Psychiatric Research. 2021;136:117-126. [cited 2021 March 20]. Available from: https://doi.org/10.1016/j.jpsychires.2021.01.054

15. Mental health of Canadians during the COVID-19 pandemic. [image on Internet]. Statistics Canada. 2020 [cited 2021 March 20]. Available from: https://www150.statcan.gc.ca/n1/en/pub/11627-m/11-627-m2020039-eng.pdf?st=L8kMkZlk

16. New research reveals impact of COVID-19 pandemic on child and youth mental health [Internet]. SickKids. 2021 [cited 2021 April 10]. Available from: https://www.sickkids.ca/en/news/archive/2021/impact-ofcovid-19-pandemic-on-child-youth-mental-health/
17. Druss B. Addressing the COVID-19 Pandemic in Populations With Serious Mental Illness. JAMA Psychiatry. 2020;77(9):891. [cited 2021 March 17]. Available from: https://doi.org/10.1001/jamapsychiatry.2020.0894

18. Yao H, Chen J, Xu Y. Patients with mental health disorders in the COVID-19 epidemic. The Lancet Psychiatry. 2020;7(4):e21. [cited 2021 Feb 22]. Available from: https://doi.org/10.1016/S22150366(20)30090-0

19. Horigian V, Schmidt R, Feaster D. Loneliness, Mental Health, and Substance Use among US Young Adults during COVID-19. Journal of Psychoactive Drugs. 2020;53(1):1-9. [cited 2021 March 20]. Available from: https://doi.org/10.1080/02791072.2020.1836435

20. Substance use and COVID-19 [Internet]. Drug Free Kids Canada. 2021 [cited 2021 March 20]. Available from: https://www.drugfreekidscanada.org/support-yourself-and-yourfamily-through-the-challenges-of-covid-19/substance-use-andcovid-19/

21. COVID-19 and Increased Alcohol Consumption: NANOS Poll Summary Report [Internet]. Canadian Centre on Substance Use and Addiction. 2021 [cited 2021 March 20]. Available from: https://www.ccsa.ca/covid-19-and-increased-alcoholconsumption-nanos-poll-summary-report

22. Aten J. Increased Screen Time and Mental Health Issues During COVID [Internet]. Psychology Today. 2021 [cited 2021 March 21]. Available from: https://www.psychologytoday.com/ca/blog/hoperesilience/202011/increased-screen-time-and-mental-healthissues-during-covid

23. Pandya A, Lodha P. Social connectedness, excessive screen time during COVID-19 and mental health: A review of current evidence. Frontiers in Human Dynamics. 2021;3. [cited 2021 November 6]. Available from: https://doi.org/10.3389/fhumd.2021.684137

24. Smith L, Jacob L, Trott M, Yakkundi A, Butler L, Barnett $Y$ et al. The association between screen time and mental health during COVID-19: A cross sectional study. Psychiatry Research. 2020;292:113333. [cited 2021 March 21]. Available from: https://doi.org/10.1016/j.psychres.2020.113333

25. What is This Much Screen Time Really Doing to Our Health? [Internet]. Harvard Pilgrim Health Care - The HaPi Guide. 2021 [cited 2021 March 20]. Available from: https://www.harvardpilgrim.org/hapiguide/what-is-this-muchscreen-time-really-doing-to-our-health/

26. Sleep and Mental Health - Harvard Health Publishing [Internet]. Harvard Health. 2021 [cited 2021 March 21]. Available from: https://www.health.harvard.edu/newsletter_article/sleep-andmental-health

27. Rafique N, Al-Asoom L, Al Sunni A, Saudagar F, Almulhim L, Alkaltham G. Effects of Mobile Use on Subjective Sleep Quality. Nature and Science of Sleep. 2020;Volume 12:357-364. [cited 2021 Feb 22]. Available from: https://doi.org/10.2147/NSS.S253375

28. Petterson S, Westfall J-M, Miller B-F. Projected Deaths of De- 
spair from COVID-19. [Internet] Providence St. Joseph Health Digital Commons. 2020;3054. [cited 2021 Aug 31]. Available from: https://digitalcommons.psjhealth.org/publications/3054

29. Starter Kit: For the National Standard of Canada for Mental Health and Well-Being for Post-Secondary Students. [Internet] Mental Health Commission of Canada. 2021. [cited 2021 March 22]. Available from: https://www.mentalhealthcommission.ca/English/media/4397

30. Mitchell T, Ortega S. Mental Health Challenges Require Urgent Response [Internet]. Inside Higher Ed. 2021 [cited 2021 Feb 22]. Available from: https://www.insidehighered.com/views/2019/10/29/studentsmental-health-shouldnt-be-responsibility-campus-counselingcenters-alone 31. Coronavirus Death Toll and Trends [Internet]. Worldometer. 2021 [cited 2021 July 21]. Available from: https://www.worldometers.info/coronavirus/coronavirusdeath-toll/ 